\section{Unpräzision in der Serie}

R.-D. Hilgers ${ }^{1}$, N. Heussen ${ }^{1}$ und S. Stanzel ${ }^{2}$

${ }^{1}$ Institut für Medizinische Statistik, Universitätsklinikum der RWTH Aachen, Aachen, Deutschland

${ }^{2}$ DKFZ Heidelberg, Heidelberg, Deutschland

\section{Englischer Begriff imprecision in series}

Definition Die Unpräzision in der Serie beschreibt die Variation der Analyseergebnisse bei mehrfacher Bestimmung aus derselben Probe innerhalb einer Serie mit demselben Messverfahren.
Beschreibung Untersucht wird dieselbe $>$ Probe in einer - Analysenserie mit denselben Reagenzien und Geräten durch denselben Analytiker unter denselben Umweltbedingungen.

\section{Literatur}

Qualitätskontrolle im Medizinischen Laboratorium von A bis Z - Ein Leitfaden in Schlagworten, 2. Aufl. Behring Diagnostika. https://doi. org/10.1515/labm.1995.19.1-12.218 